\title{
The Downside Risk of Climate Change in California’s Central Valley Agricultural Sector
}

Michael Hanemann

University of California - Berkeley

Susan Stratton Sayre

Smith College, ssayre@smith.edu

Larry Dale

Lawrence Berkeley National Laboratory

Follow this and additional works at: https://scholarworks.smith.edu/eco_facpubs

Part of the Economics Commons

\section{Recommended Citation}

Hanemann, Michael; Sayre, Susan Stratton; and Dale, Larry, "The Downside Risk of Climate Change in California’s Central Valley Agricultural Sector" (2016). Economics: Faculty Publications, Smith College, Northampton, MA.

https://scholarworks.smith.edu/eco_facpubs/2 


\title{
THE DOWNSIDE RISK OF CLIMATE CHANGE IN CALIFORNIA’S CENTRAL VALLEY AGRICULTURAL SECTOR
}

\author{
Michael Hanemann \\ University of California, Berkeley \\ Susan Stratton Sayre \\ Smith College \\ Larry Dale \\ Lawrence Berkeley National Laboratory
}

\begin{abstract}
Downscaled climate change projections for California, when translated into changes in irrigation water delivery and then into profit from agriculture in the Central Valley, show an increase in conventional measures of variability such as the variance. However, these increases are modest and mask a more pronounced increase in downside risk, defined as the probability of unfavorable outcomes of water supply or profit. This paper describes the concept of downside risk and measures it as it applies to outcomes for Central Valley agriculture projected under four climate change scenarios. We compare the effect of downside risk aversion versus conventional risk aversion or risk neutrality when assessing the impact of climate change on the profitability of Central Valley agriculture. We find that, when downside risk is considered, the assessment of losses due to climate change increases substantially.
\end{abstract}

Keywords: downside risk, climate change, variability, agriculture, outcome distribution, water resources

Hanemann's research was supported by NSF Award 1204774 to Arizona State University. Our research relies on modeling results provided to us by California Department of Water Resources and CVPM computer code provided by Steven Hatchett. We thank Sydny Fujita and Nathaniel Bush for research assistance. This draft has benefitted from the helpful suggestions of several anonymous reviewers and the associate editor. Any remaining errors are our own. 


\section{Introduction}

The Synthesis Report of the IPCC's Fourth Assessment Report famously characterized climate policy as being about managing the risks associated with possible impacts of climate change (IPCC, 2007, p. 22). Earlier commentators had also emphasized the importance of a risk perspective when assessing climate change impacts. In 1992, for example, Cline argued that an ideal impact measurement would "include some form of weighting to take account of policymaker risk aversion. If policy makers are cautious, there should be relatively more weight attributed to high-damage variants than to the low-damage variants flanking the central case" (Cline, 1992, p. 85). In California, a major pathway by which climate change could affect economic and social wellbeing is through the state's water supply, about $75 \%$ of which is used for irrigated agriculture. In this paper we examine the potential economic impact of climate change scenarios on the profitability of farming in California's Central Valley, which accounts for $75 \%$ of the state's irrigated acreage and $65 \%$ of the total value of the state's agricultural output. We explicitly adopt a risk management perspective by framing our analysis in probabilistic terms.

The risk perspective is especially appropriate for water supply. As Rayner et al. (2005) documented, water supply managers tend to be highly conservative and motivated above all to ensure that water is available when their customers need it. This concern can generate an extreme form of risk aversion on the part of water managers which goes beyond the conventional form considered in the theoretical literature; this is known as downside risk aversion. Conventional risk aversion places equal weight on unusually favorable and unfavorable outcomes. Downside risk aversion, by contrast, places a heavier weight on unfavorable outcomes than on favorable ones. The asymmetry reflects the reward structure typically faced by a water manager: he is penalized if there is too little water to meet his customers' needs, but is unlikely to receive a comparable kudos if there is more water than they need.

The implications of asymmetric rewards for outcomes in different tails of the distribution were first considered in the financial literature, where the term downside risk originated (Markowitz, 1959). ${ }^{1}$ It was based on the empirical observation that decision makers managing financial investments frequently associate risk with failure to attain a target return. ${ }^{2}$ The target return may be a positive number, or it may be zero in which case there is simply a desire to avoid a loss. With the various financial disasters of recent years, the motivation to avoid significant losses has become more salient in financial circles, and the technical literature on downside risk has grown enormously. Although the context is very different, it is likely that analogous considerations apply to water resource managers for the reasons stated above. ${ }^{3}$

\footnotetext{
${ }^{1}$ Downside risk was first modeled by Roy (1952), who noted that investors frequently put the conservation of their principal as their first goal and set some minimum acceptable return that will conserve the principal.

${ }^{2}$ See, for example, Mao (1970a,b) and Waud (1976). Some other choice situations in which downside risk aversion has been observed include pricing decisions in large companies (Lanzilotti, 1958) and peasant farming in Mexico (Masson, 1974),

${ }^{3}$ Kiparsky (2010) interviewed water managers on the eastside of the San Joaquin Valley, part of our study area, to elicit their utility functions for water supply risk and found strong evidence of downside risk aversion on their part.
} 
Following the pioneering work of Mearns et al. (1992), many researchers have included risk in their analyses of climate change and have formulated a probabilistic representation of potential impacts. But, they typically have not factored risk aversion into their analysis and they have not calculated risk premia as part of their measurement of climate damages. We not only account for risk aversion but we also introduce to the climate change literature a new form of risk aversion - downside risk aversion - that could resonate with certain decisions, including water management in California that we study here.

The paper is organized as follows. In the next section, we briefly present the climate change scenarios developed by the California Energy Commission (CEC) and the California Department of Water Resources (CDWR) for use in the Second California Scenarios Assessment (Franco et al., 2011), from which this research originated, and the translation of those climate scenarios into changes in streamflow for the rivers of the Central Valley. Section 3 applies a quadratic programming model of California agriculture, often used by CDWR and others to analyze impacts of water-related policy issues, to assess the changes in surface water allocation and resulting impacts on groundwater extraction, crop production and farm profitability in the Central Valley. Section 4 formally introduces the concept of downside risk as a tool for evaluating the change in farming profitability. The concept is applied in Section 5. Some concluding observations are presented in Section 6.

\section{Climate Change and Central Valley Water}

In the Second California Scenarios Assessment, researchers were provided with a common set of climate and sea-level rise scenarios developed by Cayan et al. (2009) derived from general circulation models (GCMs) selected for their ability to provide a reasonably accurate representation of historical climatological conditions in California. Following the Fourth Assessment Report (IPCC 2007), the A2 (medium-high) and B1 (low) emissions scenarios were used. The hydrological component of the Scenarios Assessment used the projections from the Parallel Climate Model (PCM) developed by the National Center for Atmospheric Research and the Geophysical Fluids Dynamics Laboratory Model version 2.1 (GFDL 2.1).

In each case, the GCM projections of temperature and precipitation were converted into projections of water deliveries to agricultural users in the Central Valley through three transformations: spatial downscaling, translation of downscaled climate projections into projections of runoff, and translation of runoff into projections of water deliveries. ${ }^{4}$

The downscaling used the bias corrected spatial downscaling (BCSD) method (Maurer and Hidalgo, 2008) to produce monthly projections of temperature and precipitation over 20002099 at a 1/8-degree resolution (Cayan et al., 2009). The downscaled projections were transformed by Cayan et al. (2009) into projections of runoff using the Variable Infiltration Capacity (VIC) hydrologic model (Liang et al. 1996). The projected runoffs for the end of the century (2070-2100) were used to re-scale CDWR's CALSIM-II, a network flow model which simulates the operation of the major water supply systems in the Central Valley on a monthly time step over the 82 year period 1922-2003 (Draper et al., 2004). ${ }^{5}$ This approach

\footnotetext{
${ }^{4}$ Connell-Buck et al. (2011) employ the same approach.

${ }^{5}$ CALSIM output includes stream flow for the main rivers in the Central Valley, reservoir inflows, storage,
} 
implicitly keeps the state's water infrastructure and its operation constant. ${ }^{6}$ The historical record of surface water deliveries and the rescaled projected series of deliveries constitute 82-point empirical probability distributions reflecting the year to year variability of supply under baseline conditions (1961-1990) and with climate change (2070-2099) corresponding to each emissions scenario and GCM.

Figure 1 displays those distributions. A distinctive feature is skewness with a long left tail. In baseline conditions, while the modal annual delivery is just under $14 \mathrm{MAF}$, annual deliveries fall below 10 MAF in a significant a number of years. With the PCM projections there is little change in the probability distribution of water deliveries under either emission scenario but there are some sharp changes under the GFDL projections. For the GFDL scenarios the likelihood of low delivery outcomes increases sharply.

In the next section we simulate the impact of these reduced deliveries on farming profitability in the Central Valley.

\section{Economic Impact on Central Valley Agriculture}

To assess the economic impact of policies affecting California agriculture CDWR and other state and federal agencies use a quadratic programming model which simulates market equilibrium in the markets for California's agricultural products, based on maximization of producer's plus consumer's surplus in each market (CDWR, 2008). The model assumes competitive markets in which price-taking producers maximize profit in the face of downward-sloping demand curves and subject to land and surface water constraints. It accounts for producers' choices of cropping patterns and their use of surface and groundwater. We use a version of this model, the Central Valley Production Model (CVPM), developed by Hatchett (1997) and employed by Dale et al. (2013). ${ }^{7}$

The model divides the Central Valley into 22 regions with crop production functions calibrated for each region. Each region maximizes profit by selecting the acreage to plant in alternative crops subject to a maximum acreage available. ${ }^{8}$ Given the crop demand

and releases, and water deliveries to Central Valley Project (CVP) and State Water Project (SWP) contractors throughout the Central Valley. The CALSIM projections under climate change are in Chung et al. (2009). There are also projections for mid-century (2035-2064); our analysis of those projections is presented in an online appendix. These deliveries were converted from monthly flow levels to annual deliveries based on the time in each month and matched to CVPM regions using CVPM files that match delivery nodes to CVPM regions. We modified historical non-project water deliveries by the same percentage as average changes in project water deliveries.

${ }^{6}$ In principle, some of the downside risk apparent in Figure 1 could be mitigated with additional storage capacity. The Sacramento-San Joaquin River system has only about one year's storage compared, say, to three years' storage on the Colorado River. The need for additional storage has been discussed in California for over 25 years. So far, little progress has occurred because of disagreements about who should pay for new storage. Moreover, the over-riding concern currently is for major new investment in conveyance infrastructure to replace existing conveyance facilities highly vulnerable to seismic risks.

${ }^{7}$ Other applications of CVPM and similar models include BECS (2008a,b and Medellin-Azura et al (2011)). This is a positive mathematical programming model (Howitt, 1995) calibrated to match farmers' observed cropping choices.

${ }^{8}$ CVPM includes twenty crops, some of which are aggregates of several specific crops. The twenty crops are: 
functions, crop prices respond to changes in supply conditions. ${ }^{9}$ Farmers choose to use surface water (subject to an availability constraint) or groundwater (whose cost depends on groundwater depth) and decide whether to invest in irrigation technology, assuming a constant elasticity of substitution between water effectiveness (applied water/crop demand) and irrigation capital. Farmers thus have a choice of adaptation strategy in the face of reduced water availability. ${ }^{10}$

CVPM has the capability to compute long- or short-run equilibria. In the former, farmers select crops and irrigation technology subject to their land and average water availability. In the short-run mode, designed to analyze how farmers respond to year-to-year fluctuations in water availability, farmers can vary their acreage of most crops but cannot adjust irrigation technology or acreage of permanent crops. To assess impacts on farming over the period 2070-2099, we modified the CVPM code to use the average conditions in long-run mode. Irrigation technology and permanent crop acreage were selected to maximize profits under expected future water conditions. We then conduct short-run analysis for each of the annual water delivery scenarios, freezing irrigation technology and permanent crop acreage.

Over time, reduced surface water availability will lead to increased groundwater pumping and greater depth to groundwater. To approximate these changes, we use estimates of annual reductions in groundwater levels as a function of surface water reductions from Dale et al. (2013). We compute the average reduction in surface water availability for each of the climate scenarios in the earlier parts of the century and compute the implied yearly reduction in groundwater levels. We project these yearly increases forward until the latter part of the century. We then use the climate estimates for reduced surface water availability to continue the projections through 2085, the midpoint of period 2070-2099.

CVPM is calibrated to the current economic condition of farming in California. Obviously, many factors will change during this century. We are following the standard convention in the economic literature on climate change of projecting the impacts of future climate scenarios on the current economy (e.g. Connell-Buck et al., 2011; Medellin-Azuara et al., 2011). To the extent that there is adaptation and/or endogenous technical change, our analysis overstates the climate impacts. ${ }^{11}$ Moreover, we do not consider possible changes (positive or negative) in crop yields compared to the present.

Rather than presenting results for each sub-region separately, we focus on the aggregate impact on the Central Valley as a whole, and we use the change in annual net revenue

grains (includes wheat, barley \& oats), rice, cotton, sugarbeets, corn \& corn silage, dry \& lima beans, safflower, alfalfa (includes alfalfa hay, alfalfa seed, and clover), pasture, field crops (includes sudan grass, sunflower, other misc field crops), process tomatoes, fresh tomatoes, onions \& garlic, potatoes, almonds \& pistachios, other deciduous (includes peaches, walnuts, nectarines, pears, cherries, apples, misc), subtropical (includes citrus, avocados, olives, figs, misc), vines (includes raisins, wine, and table grapes), truck crops (includes carrots, cauliflower, lettuce, peas, spinach, broccoli, asparagus, peppers, sweet potatoes, misc), and cucurb (includes cantelope, honeydew, watermelon, squash, and cucumbers).

${ }^{9}$ Output from other regions is implicitly held constant. To the extent that climate change reduces (increases) production worldwide, the price responses in the paper are under (over) estimates.

${ }^{10}$ The model does not consider the option of deficit irrigation resulting in reduced yields.

${ }^{11}$ However, adaptation can be costly, slow to occur or imperfect. Moreover, there can be some trends, such as population growth, that will increase the pressure on California's water supply as the century progresses. 
(profit) from Central Valley agriculture as the metric of impact. ${ }^{12}$

Profit is affected in several ways by the reduction in surface water supply. With less water, farmers switch away from crops with lower marginal net revenue (typically field crops) while preserving those with higher marginal net revenue (typically tree crops); less acreage is cultivated overall; there is more investment in higher-efficiency but also higher-cost irrigation; and groundwater pumping increases but this becomes more costly as the water table declines. The result is that, while aggregate profit declines, it declines less than proportionately with the decline in surface water availability or the reduction in acreage. The probability distributions of Central Valley agricultural profit at the end of the century with and without climate change are graphed in Figure 2, and the first half of Table 1 presents descriptive statistics. $^{13}$

In Figure 2, the baseline probability distribution (without climate change) is in black; the PCM distributions (low climate sensitivity) are in blue, and the GFDL distributions (medium climate sensitivity) are in red. Kolmogorov-Smirnov tests confirm that the two GFDL distributions and the PCM A2 (but not B1) distribution are statistically different from the baseline distribution. ${ }^{14}$

The existing literature (e.g., Connell-Buck et al., 2011; Medellin-Azuara et al., 2011) focuses on changes in the mean (expected value) of the distributions. In Table 1, the changes in the mean are statistically significant for the GFDL scenarios and PCM-A2 but not PCM-B1. ${ }^{15}$

However, the change in the shape of the distributions is at least as striking as the change in their central location. There is a noticeable shift of probability mass toward the left tail, reducing the degree of negative skewness. Without climate change, an annual aggregate net revenue for Central Valley agriculture below the baseline mean occurs only $37 \%$ of the time; with climate this probability increases, reaching $80 \%$ under the GFDL A2 scenario. Focusing only on the mean masks other substantial changes in the distribution of agricultural profit.

\section{Downside Risk}

There are two main strands to the literature on decision making under uncertainty, in economics and in finance. In the economics literature, the standard approach is expected utility maximization (EU): a decision maker makes risky choices ("chooses among gambles") by maximizing the expected utility of the outcome. Equivalently, he chooses the gamble with the highest certainty equivalent outcome. ${ }^{16}$ The finance literature commonly

\footnotetext{
${ }^{12}$ By contrast, Medellin-Azuara et al. (2011), who simulate just the GFDL-A2 scenario, use the change in gross revenue from agriculture as their economic metric. Gross revenue exceeds net revenue by an order of magnitude and is not generally accepted as an appropriate metric of value in economics.

${ }^{13}$ To measure skewness, we report the Fisher-Pearson coefficient and also the probability that aggregate annual profit falls below the mean value, $\$ 549.3$ million.

${ }^{14}$ The p-values are 0.00 for the GFDL scenarios, 0.010 for PCM-A2, and 0.202 for PCM-B1. In some subregions, the PCM-B1 results are also statistically different from the baseline (see online Appendix).

${ }^{15}$ The p-values are 0.0000 for the GFDL scenarios, 0.0182 for PCM-A2, and 0.1996 for PCM-B1.

${ }^{16}$ The certainty equivalent of a gamble is the outcome which, if it occurred for sure, would yield the same
} 
uses the Mean-Variance (MV) approach: choices are made by maximizing a function that depends on the mean and the variance of the outcome - the two-moment decision model. The function is increasing in the mean and decreasing in the variance (or standard deviation), the latter serving as the measure of risk. The criterion is often formulated as a linear function: ${ }^{17}$

$$
\text { Maximize } \mathrm{V}=\mu-\lambda \sigma^{2}
$$

where $\lambda>0$ is the weight placed on risk versus the expected return. If the decision maker maximizes EU and is risk-averse, the formula for $\mathrm{V}$ in (1) is a second-order approximation to the certainty equivalent for his expected utility. In some cases, it is an exact measure of the certainty equivalent. ${ }^{18}$

However, as used in (1), the variance is not always a satisfactory indicator of risk. In our case, for example, one might think that the probability distribution of agricultural profit under the GFDL-A2 scenario carries more risk of low profits and is therefore less favorable than that under GFDL-B1 (Figure 2), but GFDL-A2 actually has a smaller standard deviation than GFDL-B1 (Table 1).

To better measure risk in financial decisions where an investor might care strongly about avoiding losses, Markowitz (1959) suggested replacing the variance in (1) with the lower semivariance, $\underline{\sigma}^{2}$ (defined below), to give:

$$
\text { Maximize } V=\mu-\lambda \underline{\sigma}^{2}
$$

Markowitz felt that this optimization criterion produced better (i.e., more satisfactory) decisions but - at the time - it was harder to compute.

An EU justification for (2) was developed independently by Bawa (1975) and by Fishburn (1977). Bawa defined the lower partial moment (LPM) as a general family of below-target risk measures with parameters $\tau$ and $\alpha$ :

$$
\sigma(\tau ; \alpha)=\int_{-\infty}^{\tau}(\tau-x)^{\alpha} f(x) d x
$$

where $\tau$ is a reference level by which outcomes are assessed (e.g., a required target outcome) and $\alpha \geq 0$ is the parameter that defines the order of the moment. When $\tau=\mu$, one has the lower (i.e., below-mean) partial moments. The lower-semivariance corresponds to the case where $\tau=\mu$ and $\alpha=2: \underline{\sigma}^{2} \equiv \sigma(\mu ; 2)$. When $\alpha=0, \sigma(\tau ; 0)$ just measures the probability of an outcome below the target. When $\alpha=1, \sigma(\tau ; 1)$ measures the expected shortfall. Bawa (1975) showed that $\sigma(\tau ; \alpha)$ is mathematically related to the ordering of gambles under stochastic dominance in the cases where $\alpha=0,1$ and 2 .

Fishburn (1977) broadened the applicability of the LPM measure of risk to the case where $\alpha$ can take a fractional (positive) value. He demonstrated the equivalence of $\sigma(\tau ; \alpha)$ with stochastic dominance for all values of $\alpha \geq 0$. To this end he identified the particular utility of outcome function that would rationalize

expected utility as the gamble itself.

${ }^{17}$ Markowitz (1952); Tobin (1958). The standard deviation is sometimes used instead of the variance.

${ }^{18}$ It is an exact measure of the certainty equivalent if the gamble involves normally distributed outcomes (which is not the case here) and the decision maker has a negative exponential utility function. 


$$
\mathrm{V}=\mu-\lambda \sigma(\tau ; \alpha)
$$

as the certainty equivalent of a gamble under EU. This utility function takes the form:

$$
u(x)=\left\{\begin{array}{cc}
x & \text { for all } x \geq \tau \\
x-\lambda(\tau-x)^{\alpha} & \text { for all } x<\tau
\end{array}\right.
$$

Fishburn's formulation is known as the $\alpha-\tau$ model. The utility function in (5) exhibits what Tversky and Kahneman (1991) call loss aversion, whereby a decision maker places a greater (negative) weight on a loss than the (positive) weight he places on a gain of the same absolute magnitude - losses weigh more heavily than gains. ${ }^{19}$ Fishburn's formulation (5) is a particular case of downside risk. Fishburn showed that $\alpha<1$ implies downside riskseeking behavior, while $\alpha=1$ implies downside risk neutrality, and $\alpha>1$ implies downside risk aversion. Larger values of $\alpha$ beyond unity imply larger degrees of downside risk aversion.

The general class of utility functions associated with downside risk aversion was subsequently characterized by Menezes, Geiss and Tressler (1980) - these are utility functions, $\mathrm{u}(\mathrm{x})$, having a positive third derivative. Thus, if the decision maker is risk averse in the conventional manner (i.e., his $\mathrm{u}(\mathrm{x})$ has a negative second derivative), the distinctive feature which makes him downside risk averse is that he is less risk averse when the gamble involves larger outcome levels (larger values of $\mathrm{x}$ ).

When empirical measures of downside risk are needed, the most common approach in the literature is to use the Fishburn $\alpha-\tau$ model or some variant. Fishburn's utility function (5) implies risk neutrality for outcomes above the target level. Holthausen (1981) generalized (5) to allow for risk averse (or risk loving) preferences for above-target outcomes, extending the two-moment structure of (4) to those cases. ${ }^{20}$ In a more recent development, Ebert (2005) introduced the normalized version of the Bawa-Fishburn risk measure, defined (for $\alpha>0$ ) as:

$$
\sigma_{N}(\tau ; \alpha)=\sigma(\tau ; \alpha)^{1 / \alpha} .
$$

Ebert presented a set of axioms characterizing desirable properties for a measure of downside risk and showed that they are satisfied by this normalized index of risk. $\mathrm{He}$ suggested that it be used when setting up a mean-risk decision criterion, substituting it for $\sigma(\tau ; \alpha)$ in (4).

For the $\alpha-\tau$ model (5), Fishburn showed how to calibrate $\alpha$ and $\lambda$ for a given value of $\tau$. The parameter $\alpha$ can be calibrated by having a decision maker choose between particular gambles and a sure thing. It can also be calibrated from observed data on choices between gambles. Based on a review of empirical evidence of choices in the face of risk made by a variety of decision makers, Fishburn suggested a value of $\alpha=4$; we use that value in our main results below. ${ }^{21}$

The calibration of $\lambda$ requires observing the decision maker making some additional choices.

\footnotetext{
${ }^{19}$ Their first formulation in Kahnemann and Tversky (1979), known as prospect theory, also assumes that the utility function for downside outcomes is risk seeking, while that for upside outcomes is risk averse.

${ }^{20}$ For a further generalization, see Zakamouline (2014).

${ }^{21}$ In an online appendix, we also assess the impact of a lower level of downside risk aversion, $\alpha=2$.
} 
However, there is no single decision making entity which controls the Central Valley's irrigation water supply: decision making is diffused among several hundred retail supply agencies and multiple wholesale supply agencies. ${ }^{22}$ Therefore, there is no particular decision maker whose choices we could interrogate in order to parametrize $\lambda$. Thus, we proceed differently. We set $\lambda=1$ but, in order to adjust the relative scales of the two components in (4), we use Ebert's normalized version of Fishburn's measure of downside risk. $^{23}$ Thus, we employ the formula

$$
V=\mu-\sigma_{N}(\tau ; \alpha)=\int_{-\infty}^{\infty} x f(x) d x-\left[\int_{-\infty}^{\tau}(\tau-x)^{\alpha} f(x) d x\right]^{1 / \alpha}
$$

as the criterion function for a downside-risk-averse decision maker, as suggested by Ebert. This is not rationalized by a specific utility function, but we use it as a practical criterion for assessing risky prospects in the spirit of (4), taking the right-hand side of (7) as being like a certainty equivalent.

For the purpose of making a comparison with evaluation by a conventionally risk averse decision maker we adjust (1) by setting $\lambda=1$ and using the standard deviation instead of the variance in order to balance the scales of the two components in the same manner as in (7). Thus, for a conventionally risk averse decision maker we use the formula

$$
\mathrm{V}=\mu-\lambda \sigma \text {. }
$$

\section{Application of Downside Risk to Climate Change Impacts in the Central Valley}

In this section we apply the measures of risk just described to the distribution of changes in agricultural profitability in the Central Valley graphed in Figure 2. We assess the impact of climate change on net revenue in three ways. First, we compute the change in expected net revenue, which measures how a risk neutral individual would view the impact of climate change. Then we compute the certainty equivalent value of net revenue for a conventionally risk averse decision maker using the formula in (1). Finally we compute the certainty equivalent value of net revenue for a downside risk averse decision maker using the certainty equivalent formula based on Ebert's normalized risk measure, (7).

Table 1 presents the results of these calculations. The first thing to note is that, while the climate change scenario reduces agricultural net revenue in every case, in three of the four cases the reduction in the certainty equivalent value of net revenue for a conventionally risk averse decision maker is smaller - both absolutely and proportionally -- than that for a risk neutral decision maker. In the case of emission scenario A2 as projected by GFDL, for example, expected net revenue declines by $26 \%$ whereas the certainty equivalent value for a conventionally risk averse decision maker declines by only $10 \%$. The increase in variance is small relative to the decrease in mean. However, the increase in the downside variance is large, and the reduction in the downside risk certainty equivalent is substantial compared to the reduction in both expected value and conventional risk certainty equivalent. The reduction in the certainty equivalent value for a downside risk decision maker is more than twice as large as for a conventionally risk averse decision maker in the case of PCM-A2

\footnotetext{
${ }^{22}$ Many of the individual irrigation districts have their own surface water rights and/or pump groundwater in addition to receiving water from wholesale agencies.

${ }^{23}$ In an online appendix, we perform a sensitivity analysis using values of $\lambda=0.5$ and $\lambda=1.5$.
} 
and GFDL-B1, and almost six times as large in the case of GFDL-A2. And, the reduction in the certainty equivalent value for a downside risk averse decision maker is twice as large as the reduction in expected value, the metric typically used in existing analyses of the economic impact of climate change. These results demonstrate the potential significance of downside risk for decision makers.

The results inevitably depend on the specific parametrization employed in representing both conventional risk aversion and downside risk aversion. As a robustness check, we also employ some other parameter values: $\alpha=2$ instead of Fishburn's suggested value of $\alpha=4$, which makes the decision maker less downside risk averse, and $\lambda=0.5$ or $\lambda=1.5$, which alternatively lowers or raises the weight placed on the index of downside risk. The full set of these results is presented in a table posted in the online Appendix. In every case, we obtain qualitatively similar results: the reduction in the certainty equivalent value for a downside risk decision maker is substantially larger than the reduction in the expected value of net return, and is more than twice as large as the reduction in the certainty equivalent value for a conventionally risk averse decision maker. Thus, to the extent that water managers and other policymakers may be concerned about downside risk, reporting impacts based on expected net revenue or on conventional mean-variance certainty equivalent would understate the likely impact of climate change.

\section{Conclusion}

In this paper, we have made two contributions to the literature on the economic impacts of climate change. First, we have modeled the economic impact of climate change on the profitability of farming in California's Central Valley probabilistically, using a discretized representation of the probability distribution of net revenue under both baseline and climate change conditions. Conducting a probabilistic analysis involves considerably more computation than performing an analysis based on the change in the expected value of the outcome, which what is usually done in the economic literature on climate change impacts. The economic modeling analysis has to be repeated for each point in the discretized representation of the probability distribution as opposed to being done just once with the expected value. Using an 82-point representation of the probability distribution of outcomes, we simulated market equilibrium in the markets for California's agricultural commodities at each discrete point of the probability distribution, both with and without climate change. The result is a richer and more nuanced representation of how California agriculture might fare under climate change.

The most visible and striking feature, as shown in Figures 1 and 2, is an increase in downside risk with regard both to the availability of surface water for irrigation and farm profitability in the Central Valley. Our second contribution, intended to account for this, was to introduce and apply the concept of downside risk. We measured a downside risk averse decision maker's certainty equivalent value for the probability distributions of net revenue before and after climate change, and found that accounting for downside risk aversion significantly raises the estimated impact of the climate change scenarios. 